\title{
Life Satisfaction and Social and Emotional Support Among Asian American Older Adults
}

\author{
Riti Shimkhada, PhD, Hin Wing Tse, BA, and Ninez A. Ponce, PhD
}

Background: Little data exist on the well-being of older adults from Asian American (AA) communities.

Methods: Using data from the 2018 California Health Interview Survey, we examined 2 well-being metrics among AAs and AA subgroups (Korean, Filipino, Vietnamese, Chinese) 65 years and older.

Results: AA older adults reported lower life satisfaction and not having needed social and emotional support compared with all other race/ethnicities. Current life satisfaction among AA older adults was $54 \%$ compared with $80 \%$ for all other race/ethnicities; $56 \%$ of AA older adults reported usually or always receiving social/emotional compared with $80 \%$ for all other race/ethnicities. Within the AA category, life satisfaction was $\mathbf{4 0 \%}$ for Korean, $\mathbf{4 8 \%}$ for Chinese, $\mathbf{4 7 \%}$ for Vietnamese, and $\mathbf{7 7 \%}$ for Filipino older adults. Among Korean older adults, 30\% reported receiving needed social/emotional support, 57\% among Chinese, 59\% among Filipino, and 65\% among Vietnamese older adults.

Conclusion: AA older adults report lower life satisfaction and not needed receiving social and emotional support compared with all other race/ethnicities. Among AAs, Korean older adults were most likely to report poorer well-being. AA older adult communities may be in urgent need of further research and investment in interventions. ( $\mathrm{J}$ Am Board Fam Med 2022;35:203-205.)

Keywords: Aging, Asian Americans, California, Health Surveys, Mental Health, Minority Health, Personal Satisfaction, Primary Health Care

\section{Introduction}

Little data exist on the well-being of older adults from Asian American (AA) communities. We report here on 2 well-being metrics-life satisfaction and receiving needed emotional support-among Chinese, Filipino, Korean, and Vietnamese older adults 65 years and older. Well-being metrics offer an opportunity to gauge how people feel about their lives, distinct from conventional health status measures. ${ }^{1}$

\section{Methods}

We used the California Health Interview Survey (CHIS) 2018 well-being measures for older adults 65 years and older to examine well-being among

This article was externally peer reviewed.

Submitted 2 June 2021; revised 26 August 2021; accepted 9 September 2021.

From UCLA Center for Health Policy Research, Los Angeles (RS, HWT, NP); UCLA Fielding School of Public Health, Department of Health Policy and Management, Los Angeles (NP).

Funding: none.

Conflict of interest: none.

Corresponding author: Riti Shimkhada, PhD, UCLA Center for Health Policy Research, 10960 Wilshire Blvd \#1550, Los Angeles, CA 90024 (E-mail: riti@ucla.edu).
AAs and AA subgroups in California. CHIS is the nation's largest state-level population-based health survey, with about 20,000 interviewed annually. Race/ethnicity groups are defined by the US Office of Management and Budget single race categories. Weighted estimates are presented here.

We examine 2 specific measures of well-being: high current life satisfaction (reporting 7 or higher on a scale of 0 to 10) and always or usually receiving needed social and emotional support (response categories to the question include always, usually, sometimes, rarely, never). Statistical comparisons were made between AAs and other race/ethnic groups and within AA subgroups. Our sample consists of 8158 individuals 65 years and older in CHIS 2018. We present here notable disparities in well-being measures for AAs and AA subgroups-Korean, Filipino, Vietnamese, and Chinese-65 years and older.

\section{Results}

We found $56 \%$ of AA older adults reported usually or always receiving social/emotional support (Table 1), compared with $80 \%$ aggregated across all non-AA groups, significantly different at the $P<.05$ 
Table 1. Social Emotional Support and Current Life Satisfaction Among Adults, 65 Years and Older, California Health Interview Survey, 2018*: Usually or Always Receiving Needed Social and Emotional Support and Current Life Satisfaction 7 or Above on a Scale of 0-10

\begin{tabular}{lrr}
\hline & & \% (95\% Confidence Interval) \\
\cline { 2 - 3 } & Social/Emotional Support & Current Life Satisfaction \\
\hline Asian American (AA) & $56.3(47.5$ to 65.2$)$ & $53.9(46.7$ to 61.1$)$ \\
Chinese & $56.7(44.2$ to 69.2$)$ & $48.0(35.5$ to 60.6$)$ \\
Korean & $29.8(14.9$ to 44.7$)$ & $39.7(22.0$ to 57.5$)$ \\
Filipino & $58.7(39.4$ to 78.0$)$ & $76.6(56.5$ to 96.7$)$ \\
Vietnamese & $65.3(49.3$ to 81.4$)$ & $47.2(29.6$ to 64.8$)$ \\
All Non-AA & $79.8(77.2$ to 82.4$)$ & $70.0(78.0$ to 82.1$)$ \\
Black & $66.4(52.1$ to 80.8$)$ & $74.3(55.6$ to 85.0$)$ \\
Latino & $72.0(65.6$ to 78.4$)$ & $82.7(80.5$ to 84.9$)$ \\
White & $83.3(81.1$ to 85.5$)$ & \\
\hline
\end{tabular}

*Due to statistical instability the following groups are not shown in the table: Native Hawaiian/Pacific Islander, American Indian/ Alaska Native, two or more races, South Asian, Japanese, other Asian; race/ethnicity is defined by U.S. Office of Management and Budget categories, which include single race and ethnicity categories (eg, AA is single race, non-Latino); there were no significant differences in poverty (under $200 \%$ federal poverty level), living alone (1 in household), \% married, years lived in United States (10+ years), age, place of residence (urban, rural, suburban) between any of the $65+$ AA subgroups.

significance level. Among AA disaggregated populations, we found that Korean older adults reported significantly lower social/emotional support (30\%) compared with Chinese (57\%) and Filipino (59\%) older adults $(P<.05$ level).

As with social/emotional support, we found AA older adults (54\%) reported significantly lower levels of life satisfaction than all other non-AA older adults (80\%). Looking at the disaggregated AA subgroups, we found Korean older adults had lower reports of life satisfaction than the other AA subgroups but not significantly different at the $P<.05$ significance level.

\section{Discussion}

We found levels of social and emotional support and life satisfaction among AA older adults in California in 2018 were lower than non-AA populations. Prior work on social isolation and loneliness suggests family physicians and other primary care providers are often the only point of social contact for many older adults, thus, just as physicians screen for other risk factors, physicians may consider paying attention to identifying patients who need emotional support and recommend to them ways to strengthen social connections. ${ }^{2}$ While primary care providers may be the key to identifying older adults who have low social and emotional support, there is a recognized dearth of evidence-based interventions on improving social isolation and loneliness in older adults, ${ }^{3}$ making it difficult for clinicians to refer patients in need of support. Nonetheless, clinicians may still be key to offering access to resources that do exist. For instance, in light of rising Asian hate crimes during the COVID-19 pandemic, it has been proposed that clinicians might spend time in conversations with older adults to identify psychological strain and offer resources to those who need them from community organizations, social workers, case managers, or psychiatrists. ${ }^{4}$ Other research points to the importance of community-based mental health screening and support groups, such as at temples, churches, and community centers, for AA older adult communities. ${ }^{5}$

While our research is unable to establish if wellbeing among AA older adults increased or decreased since the COVID-19 pandemic, it is plausible that the rise in hate crimes targeted toward AA older adults has raised anxiety in the community. There have been a number of community-based initiatives across the United States involving volunteers chaperoning older adult AAs in their neighborhoods, providing safety and companionship. It remains to be seen if these efforts bloom into programs that invite longer-term emotional support into the lives of AA older adults. Communities may be in more urgent need of further research and investment in interventions, including those that involve primary care providers and those that seek to understand the systemic 
and structural barriers facing AA older adults that lead to low levels of social/emotional support and life satisfaction.

To see this article online, please go to: http://jabfm.org/content/ 35/1/203.full.

\section{References}

1. Stiefel MC, Gordon NP, Wilson-Anumudu FJ, Arsen EL. Sociodemographic determinants of health and well-being among adults residing in the combined Kaiser Permanente regions. Perm J 2019;23:18-91.
2. Freedman A, Nicolle J. Social isolation and loneliness: the new geriatric giants: approach for primary care. Can Fam Physician 2020;66:176-82.

3. Blazer D. Social isolation and loneliness in older adults-a mental health/public health challenge. JAMA Psychiatry 2020;77:990-1.

4. Lee JH. Combating Anti-Asian sentiment-a practical guide for clinicians. N Engl J Med 2021;384:2367-9.

5. Oh H, Ko J, Waldman K. Church-based sleep screenings to detect mental health problems among Korean-Americans. J Immigr Minor Health 2019; 21:1440-3. 